\title{
Molde de silicone baseado em dentição natural: técnica para mimetizar a textura superficial dentária em resina composta
}

\section{Silicone index based on natural dentition: technique to mimic the dental surface texture in composite resin}

\author{
Luiz Gustavo Fernandes Lima Oliveira* \\ Marlus da Silva Pedrosa* \\ Leticia de Sá Evelin ${ }^{* * *}$ \\ José Guilherme Férrer Pompeu ${ }^{* * *+*}$
}

\section{Resumo}

A antevisão do tratamento definitivo é um dos aspectos essenciais em planejamentos envolvendo a estética dentária, e os maiores desafios envolvem a realização de texturas de superfície naturais e realistas e a previsibilidade dos resultados finais. Objetivo: apresentar uma técnica que utiliza molde de silicone baseado em dentição natural a fim de mimetizar a textura superficial dentária. Materiais e método: pré-molares extraídos para fins ortodônticos foram utilizados para confecção de uma matriz de silicone de adição. Uma resina composta fluida fotopolimerizável foi utilizada para produzir conchas de resina capazes de duplicar a forma e a textura das coroas dos dentes naturais. Conclusão: a técnica descrita proporcionou a obtenção de cascas anatômicas com formas e texturas naturais, que podem ser utilizadas como alternativa prática à realização de restaurações provisórias diretas intraorais ou no auxílio ao enceramento.

Palavras-chave: Dentição. Estética dentária. Materiais dentários. Resinas compostas.

\section{Introdução}

A odontologia, nos últimos anos, tem aumentado o seu campo de atuação ${ }^{1}$, e pacientes estão cada vez mais buscando a experiência de cirurgiões-dentistas para realização de procedimentos restauradores estéticos ${ }^{2}$. Os maiores desafios em odontologia estética envolvem a realização de texturas de superfície naturais e realistas, bem como a previsibilidade dos resultados finais ${ }^{3}$.

Em casos envolvendo a estética dentária, a antevisão e o acurado planejamento, são necessários para garantir o mais adequado resultado ${ }^{4,5}$. Mudanças estéticas ou funcionais são melhor experimentadas com restaurações provisórias, de modo que as modificações possam ser facilmente realizadas intraoralmente ou quando satisfatoriamente copiadas para as restaurações definitivas ${ }^{6}$.

O enceramento diagnóstico, técnica tradicionalmente utilizada por muitos profissionais da odontologia ${ }^{7}$, e a confecção de restaurações provisórias, quando adequadamente fabricadas, permitem melhor planejamento e determinação dos requisitos estéticos e funcionais de procedimentos restauradores finais ${ }^{6,8,9}$

A mimetização e a reprodução da textura da superfície de dentes naturais guiam a concepção de boas restaurações. Assim, de acordo com o conceito no qual conchas de resina composta (Anatomic Shell

Clínica Privada, Luiz Gustavo Odontologia Estética e Implantodontia, Teresina, Piauí, Brasil.

Graduado em Odontologia, Faculdade Integral Diferencial, Teresina, Piauí, Brasil.

Mestranda em Reabilitação Oral, Faculdade de Odontologia de Ribeirão Preto, Ribeirão Preto, São Paulo, Brasil.

Professor titular, Departamento de Odontologia Restauradora, Universidade Federal do Piauí, Teresina, Piauí, Brasil. 
Technique) foram utilizadas para fornecer texturas realistas ao resultado estético final ${ }^{3}$, o presente trabalho objetivou apresentar uma técnica alternativa de reconstrução coronal provisória, que utiliza molde de silicone baseado em dentição natural, a fim de mimetizar a textura superficial dentária.

\section{Materiais e método}

Como forma de contemplar objetivos estéticos, práticos e satisfatórios, bem como incorporar naturalidade na etapa de planejamento do tratamento reabilitador, o presente trabalho descreve uma técnica de reconstrução coronal baseada em dentição natural. Para tal, pré-molares (Figuras 1 e 2) extraídos para fins ortodônticos, limpos e previamente esterilizados, adquiridos mediante assinatura de termo de doação pelo paciente, foram utilizados como modelos para obtenção de moldes de silicone.

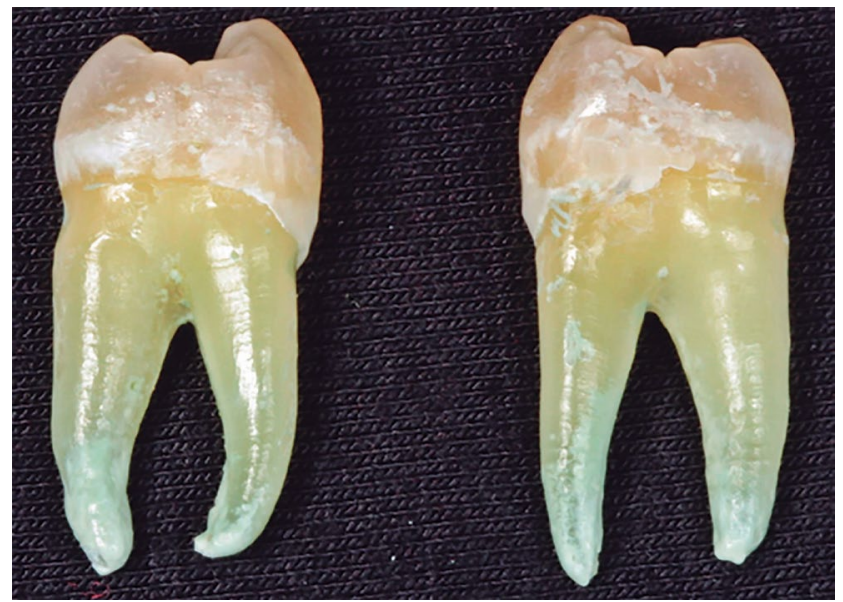

Figura 1 - Superfície proximal dos pré-molares

Fonte: elaboração dos autores.

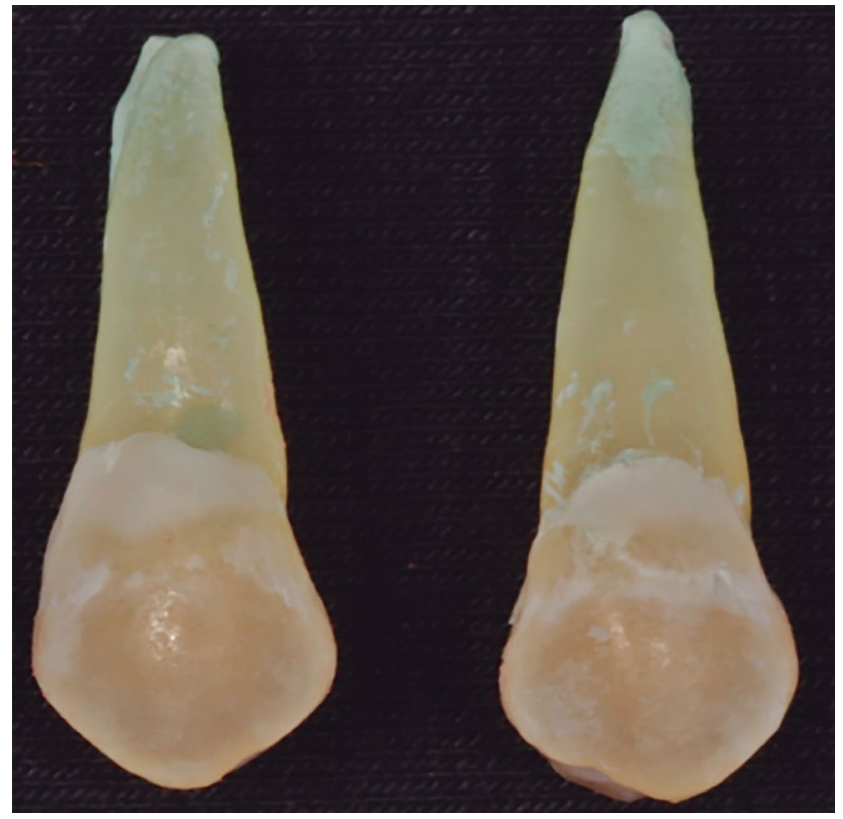

Figura 2 - Superfície vestibular dos pré-molares

Fonte: elaboração dos autores.
A matriz foi confeccionada com utilização de silicone de adição, segundo a técnica de moldagem em dois tempos. A pasta pesada do material de moldagem Virtual Putty Regular (Ivoclar Vivadent, Schann, Liechtenstein, lote: UL2353) foi previamente manipulada conforme as especificações do fabricante, no tocante à proporção da pasta pesada/ catalisador, e acomodada em um recipiente plástico, com as dimensões: $17 \mathrm{~mm}$ x $17 \mathrm{~mm}$ x $24 \mathrm{~mm}$. Sob leve pressão digital, os dentes foram adaptados com sua face vestibular voltada para o material de moldagem (Figura 3).

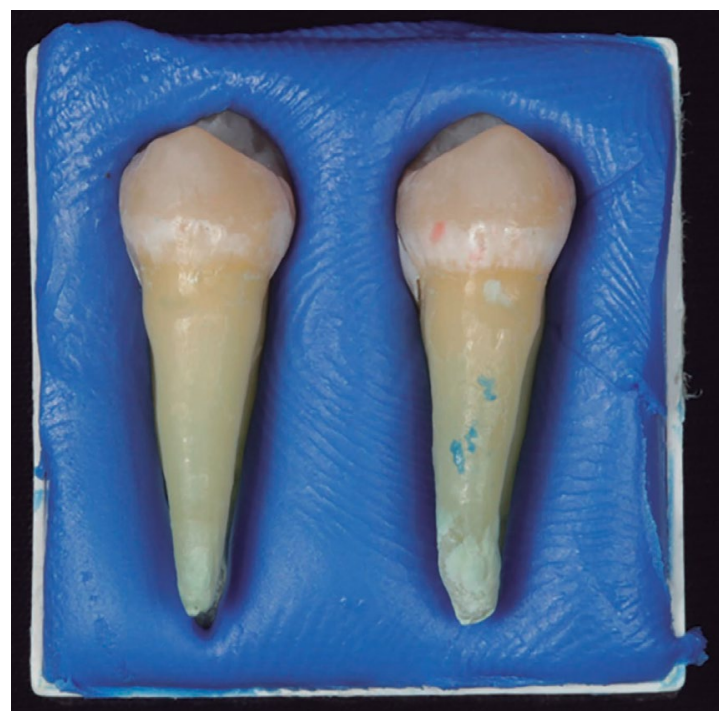

Figura 3 - Adaptação da superfície vestibular dos dentes na matriz de silicone

Fonte: elaboração dos autores.

Após a presa final do material de moldagem, o dente foi cuidadosamente removido do interior da matriz (Figura 4). O silicone de adição Virtual Light Body Regular (Ivoclar Vivadent, Schann, Liechtenstein, lote: UL2316) foi acomodado com auxílio de um dipensador pistola universal (Sulzer Mixpac, Suiça, lote: 2180141) (Figura 5).

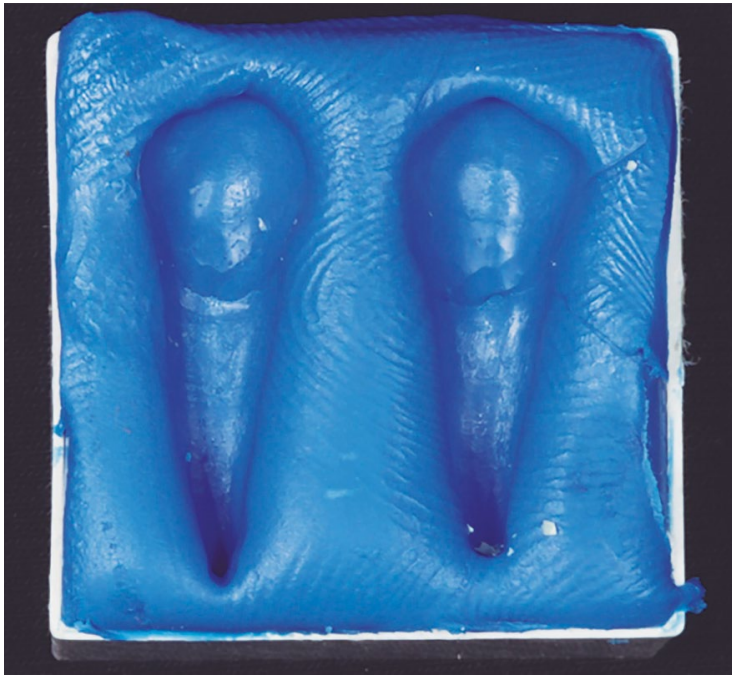

Figura 4 - Matriz com dente removido do seu interior Fonte: elaboração dos autores. 


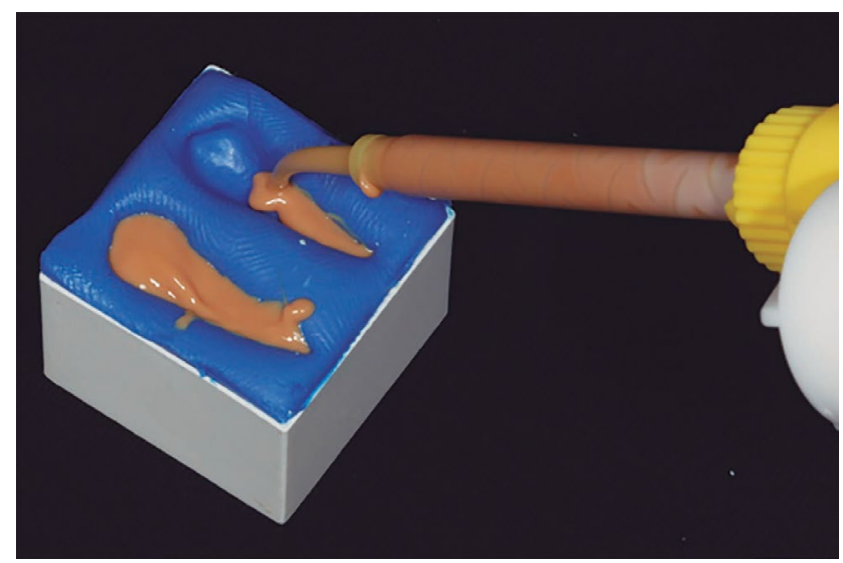

Figura 5 - Silicone de adição Virtual Light Body Regular acomodado no interior da matriz

Fonte: elaboração dos autores.

Adaptou-se novamente os dentes sobre o material de moldagem (Figura 6) e aguardou-se a presa completa do material. Os dentes foram removidos do interior da matriz (Figura 7). A matriz confeccionada foi cuidadosamente recortada com lâmina de bisturi de aço carbono descartável n. 15 (Maxicor, Paraná, Brasil, lote: 140518), para não danificar a região de interesse correspondente à face vestibular do dente (Figura 8).

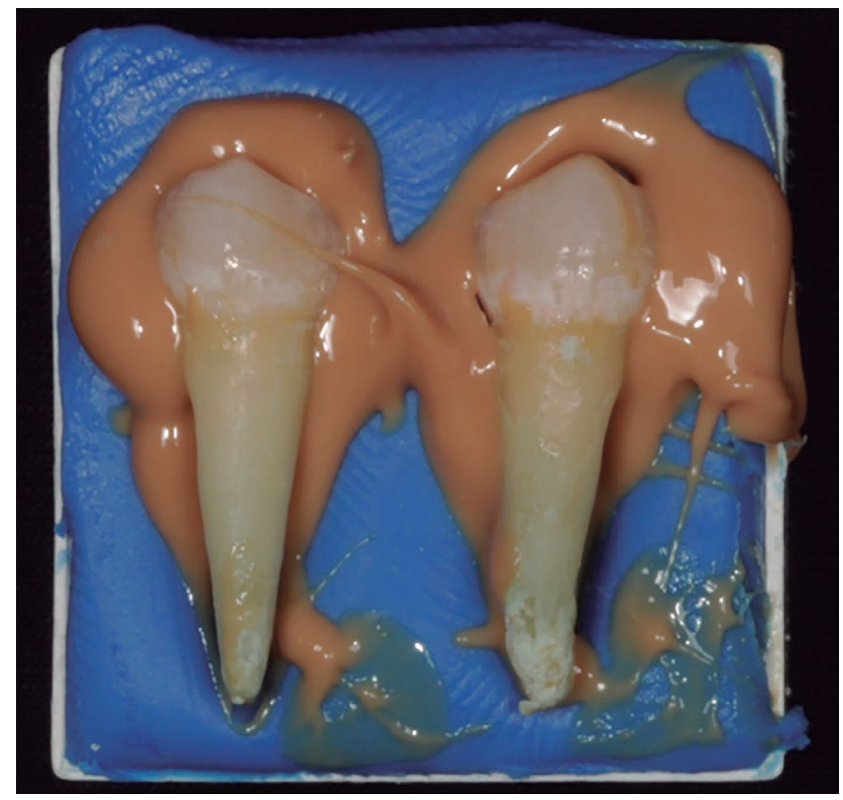

Figura 6 - Adaptação dos dentes sobre o material de moldagem Fonte: elaboração dos autores.

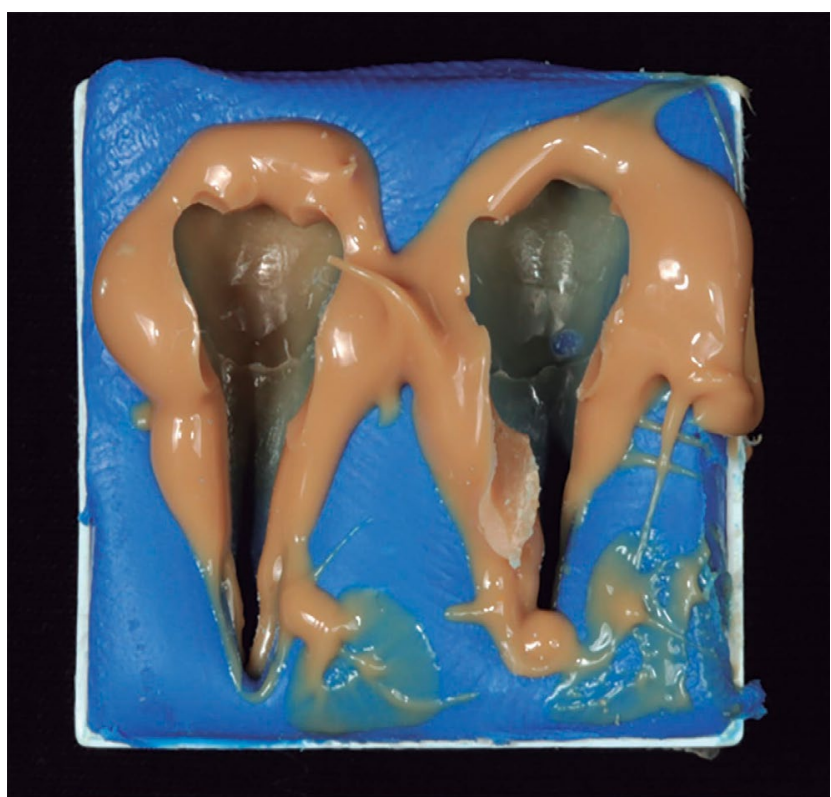

Figura 7 - Dentes removidos do interior da matriz

Fonte: elaboração dos autores.

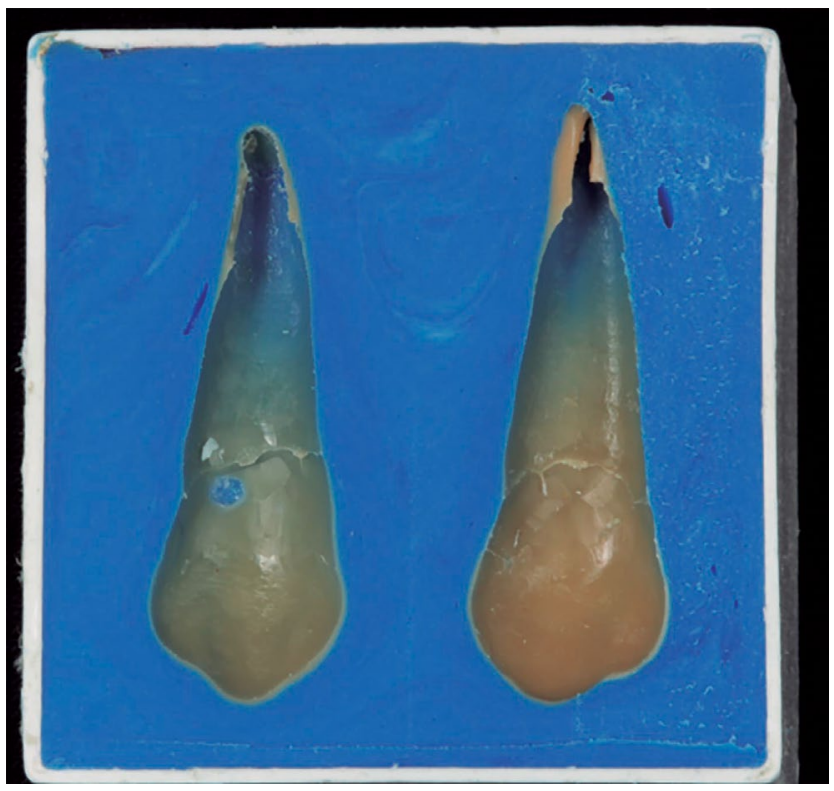

Figura 8 - Matriz confeccionada após acabamento

Fonte: elaboração dos autores.

Uma resina composta fluida fotopolimerizável Natural Flow (Nova DFL, Rio de Janeiro, Brasil, lote: 16070908) foi dispensada na região correspondente à coroa dentária da matriz de silicone confeccionada (Figura 9). Após fotopolimerização completa do material resinoso, com fotopolimerizador LED de amplo espectro (Valo, Ultradent, Brasil, lote: C33887), com potência de $3.200 \mathrm{~mW} / \mathrm{cm}^{2}$ por três segundos (Figura 10), foram produzidas conchas de resina capazes de duplicar a forma e a textura da face vestibular dos dentes naturais (Figura 11). 


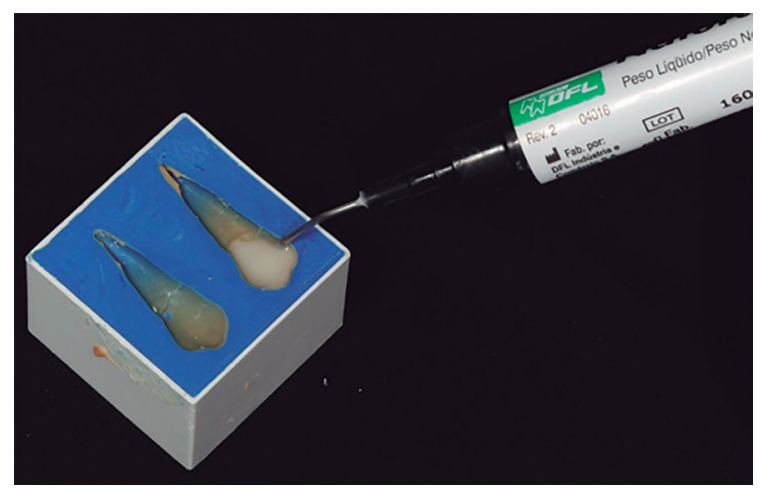

Figura 9 - Resina composta fluida dispensada na região correspondente à coroa dentária da matriz de silicone confeccionada

Fonte: elaboração dos autores.

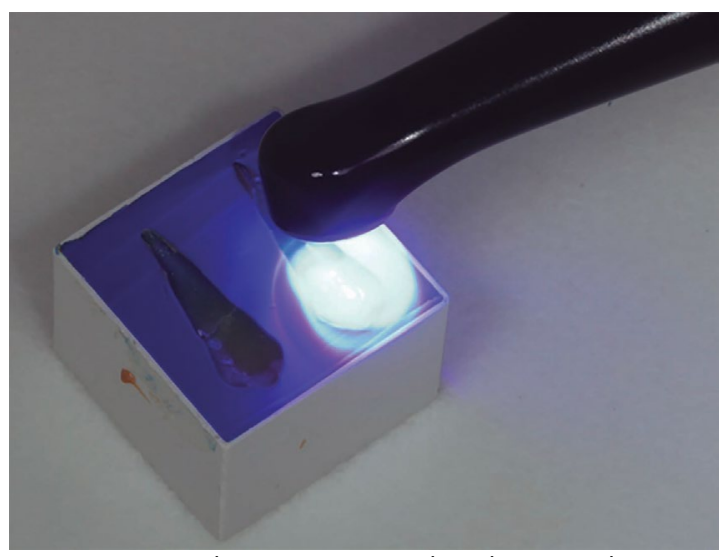

Figura 10 - Fotopolimerização completa do material resinoso Fonte: elaboração dos autores.

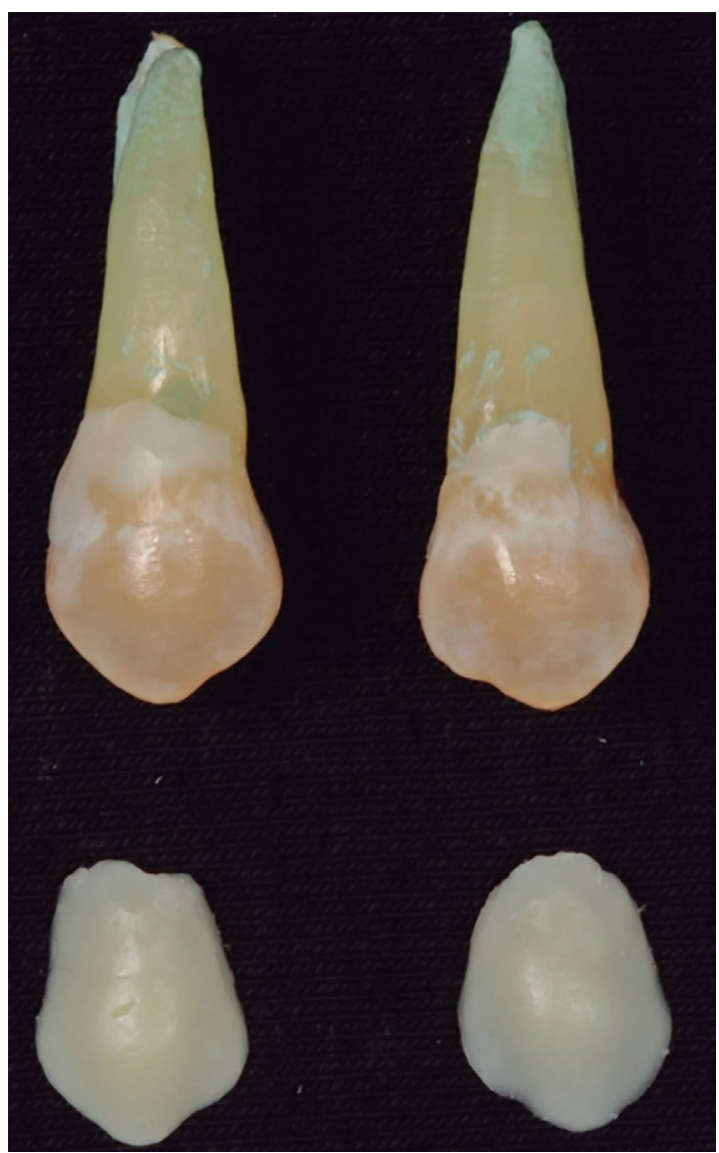

Figura 11 - Conchas de resina produzidas

Fonte: elaboração dos autores.
As conchas produzidas foram provadas sobre as superfícies vestibulares dos dentes correspondentes no modelo de estudo. Para fixação da concha de resina, dispensou-se uma pequena quantidade de resina fluida fotopolimerizável Natural Flow (Nova DFL, Rio de Janeiro, Brasil, lote: 16070908) na região central do dente correspondente no modelo de estudo, seguida de sua adaptação (Figura 12) e posterior fotopolimerização por 3 três segundos, com fotopolimerizador LED de amplo espectro (Valo, Ultradent, Brasil, lote: C33887), com potência de $3.200 \mathrm{~mW} / \mathrm{cm}^{2}$.

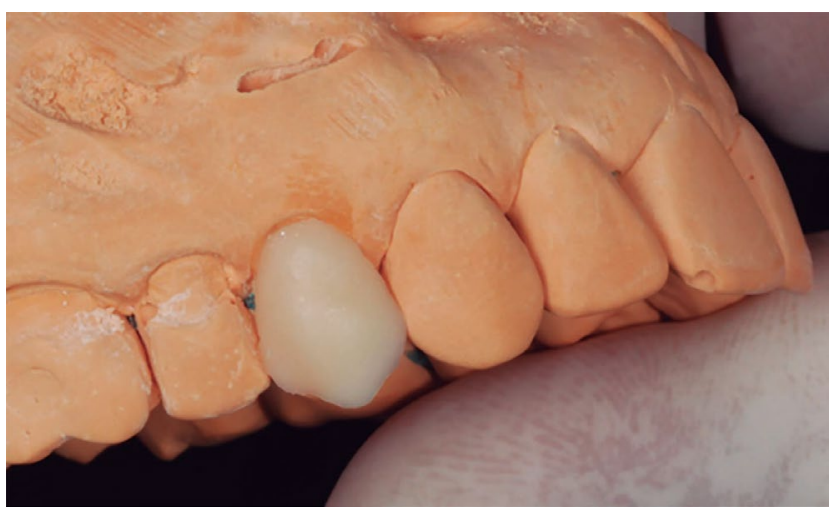

Figura 12 - Adaptação das conchas de resina na superfície correspondente do modelo

Fonte: elaboração dos autores.

Procedeu-se com aplicação de resina fluida na região oclusal correspondente à interface dente/concha e posterior fotopolimerização (Figura 13). A Figura 14 mostra o caso finalizado em dez elementos dentários, segundo a técnica descrita. Para cada um dos elementos, utilizou-se um molde de silicone baseado na dentição natural do dente correspondente.

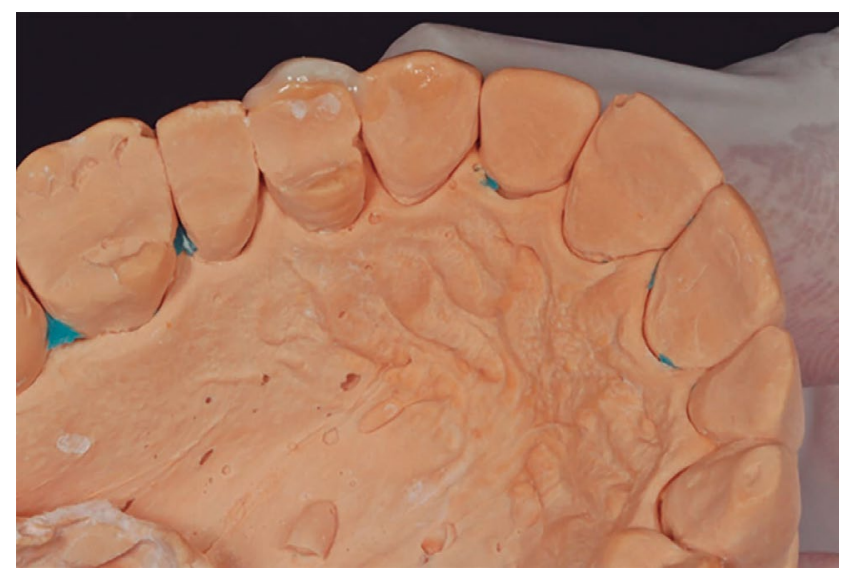

Figura 13 - Aplicação de resina fluida na região oclusal correspondente à interface dente/concha e posterior fotopolimerização

Fonte: elaboração dos autores. 


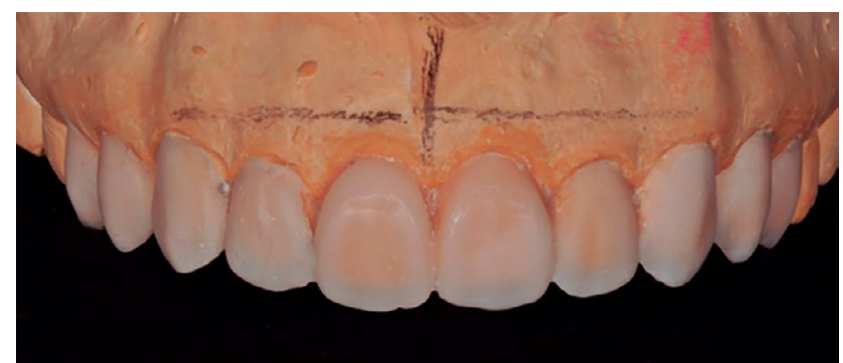

Figura 14 - Modelo com dez elementos dentários concluídos segun do a técnica descrita

Fonte: elaboração dos autores.

\section{Discussão}

A estética, atualmente, é um ponto-chave em diversas especialidades da odontologia, uma vez que os pacientes estão muito mais conscientes da atratividade de um belo sorriso ${ }^{10,11}$. Quando mudanças funcionais e estéticas são visadas, torna-se essencial a premeditação e o planejamento, para garantir o tratamento reabilitador mais adequado. Os profissionais de odontologia estão cada vez mais aderindo a ensaios restauradores (mock-ups odontológicos) como forma de vislumbrar o resultado do planejamento final. Esses ensaios restauradores podem ser obtidos a partir do enceramento de modelos de estudo ${ }^{12-15}$.

O enceramento como auxílio para o diagnóstico no planejamento do tratamento odontológico tem sido aplicado em várias situações, desde a simples avaliação do paciente até reabilitações orais extensas $^{16}$. Além disso, representa um elemento-chave em procedimentos cirúrgicos e restauradores, pois permitem visualizar o resultado final projetado ${ }^{17}$, com ou sem a realização de ensaios restauradores intraorais guiados, bem como contribuem para a confecção de restaurações de caráter provisório.

Diversas técnicas envolvendo uma grande variedade de materiais estão disponíveis para confecção de restaurações satisfatórias, as quais podem ser realizadas diretamente sobre a superfície dos dentes ${ }^{18}$ ou indiretamente, por meio de moldagem ${ }^{19}$, além da possibilidade de combinação de técnicas diretas e indiretas. Ressalta-se que os profissionais precisam estar familiarizados com a gama de materiais e técnicas disponíveis.

A mimetização e a reprodução da textura da superfície do dente natural poderiam guiar a concepção de uma boa restauração estética sem a necessidade de um técnico em prótese dentária altamente qualificado ${ }^{3}$. Desenvolvido por Paul Kano e Lívio Yoshinaga ${ }^{20}$, o conceito Skyn permite a redução de etapas laboratoriais importantes, permitindo confeccionar laminados, coroas ou uma reabilitação oral completa, totalmente individualizada e personalizada para cada paciente. Considerando-se que a natureza é exuberante na morfologia, a utilização desse conceito permite a confecção das restaurações em inúmeras possibilidades ${ }^{20}$.

Os modelos de Hajto são réplicas da dentição natural anterior, com exemplos de diferentes for- mas, tamanhos e texturas de superfície dentária ${ }^{3}$. O presente trabalho utilizou moldes de silicone, previamente confeccionados a partir de pré-molares, para criar cascas anatômicas com formas e texturas naturais, capazes de serem utilizadas como alternativa prática à realização de restaurações provisórias diretas intraorais ou ao enceramento diagnóstico.

A técnica descrita pode ser vista como uma alternativa à utilização dos modelos Hajto e similares para a confecção das chamadas conchas de resina composta, pois apresenta viabilidade de uso por profissionais com pouca experiência prática na realização de enceramentos diagnósticos ou restaurações com formas e texturas naturais.

Nesse sentido, o profissional pode utilizar dentes naturais com coroas intactas, extraídos por razões diversas, tais como para fins ortodônticos, periodontais ou por fraturas e reabsorções dentárias, para confeccionar moldes de silicone que poderão ser empregados em reconstruções coronárias. Alguns aspectos devem ser levados em consideração ao se utilizar essa técnica, tais como a necessidade de ajustes para adaptação e a precisão marginal das conchas de resinas quando aplicadas nos modelos de estudo ou em superfícies dentárias, bem como o respeito aos aspectos éticos referentes à utilização de dentes humanos. Os moldes de silicone também podem ser empregados em técnicas semidiretas para confecção de facetas de resina composta.

\section{Conclusão}

Por meio das conchas de resina confeccionadas com a utilização da técnica de reconstrução coronal provisória que utiliza molde de silicone baseado em dentição natural, foi possível mimetizar a textura da superfície dentária. Essa técnica pode ser empregada como alternativa para realização de enceramentos diagnósticos para ensaios restauradores bem como para confecção de restaurações provisórias estéticas.

\section{Abstract}

The anticipation of definitive treatment is one of the essential aspects in dental aesthetic planning in which the greatest challenges involve the realization of natural and realistic surface textures, as well as to ensure predictability to the final results. Objective: this article presents a technique that employs silicone index based on natural dentition in order to mimic and reproduce the natural tooth surface and texture. Materials and method: premolars extracted for orthodontic purposes were used to make an addition silicone index. A lightcured composite resin was used to produce resin shells capable of doubling the shape and texture of natural tooth crowns. Conclusion: the described technique provided the obtaining of anatomical shells with natural shapes and textures capable of being used as a practical alternative the realization of direct intraoral restorations or auxiliary to waxing.

Keywords: Dentition. Esthetics dental. Dental materials. Composite resins. 


\section{Referências}

1. Carvalheira TB, Goyatá FR, Rodrigues CRT, Souza MCA. Resolução estética em dentes anteriores com coroas totais livres de metal - relato de caso clínico. Int J Dent 2010; 9(2):102-6.

2. Basso KCFJ, Pedro FLM, de Barros YBAM, da Silva MB, Tavarez RRJ, de Andrade MF, et al. Planning and clinical strategy in direct composite restorations. Sci J Dent 2014; 1:35-7.

3. Silva NRFA, Kano P, Van Dooren E, Xavier C, Ferencz JL, Lacerda E. Improving aesthetics in CAD/CAM dentistry anatomic shell technique. Industry Report 2003; 2:30-3.

4. Calixto LR, Bandeca MC, Adrade MF. Enceramento diagnóstico: previsibilidade no tratamento estético indireto. Rev Dental Press Estet 2011; 8(3):26-37.

5. Gouveia THN, Theobaldo JD, Vieira-Junior WF, Lima DANL, Aguiar FHB. Esthetic smile rehabilitation of anterior teeth by treatment with biomimetic restorative materials: a case report. Clin Cosmet Investig Dent 2017; 11(9):27-31.

6. Astudillo-Rubio D, Delgado-Gaete A, Bellot-Arcís C, Montiel-Company JM, Pascual-Moscardó A, Almerich-Silla JM. Mechanical properties of provisional dental materials: A systematic review and meta-analysis. PLoS One 2018; 13(2):e0193162.

7. Seymour DW, Patel M, Chan MFWY. Aesthetic preview: a novel approac. Dent Update 2012; 39:422-6.

8. Keys WF, Keirby N, Ricketts DNJ. Provisional restorations a permanent problem? Dent Update 2016; 43(10):908-12.

9. Kozmacs C, Baumann V, Bunz O, Piwowarczyk A. Relative clinical success of bis-acryl composite provisional crowns. Compend Contin Educ Dent 2018; 39(1):e9-e12.

10. Al-Shahrani I. Perception of professional female college students towards Smile arc Types and Outlook about their appearance. J Int Soc Prev Community Dent 2017; $7(6): 329-35$.

11. Deng X, Wang YJ, Deng F, Liu PL, Wu Y. Psychological well-being, dental esthetics, and psychosocial impacts in adolescent orthodontic patients: a prospective longitudinal study. Am J Orthod Dentofacial Orthop 2018; 153(1):87-96.e2.

12. Faus-Matoses V, Faus-Matoses I, Jorques-Zafrilla A, Faus-Llácer VJ. Orthodontics and veneers to restore the anterior guidance. A minimally invasive approach. J Clin Exp Dent 2017; 19(11):e1375-e8.

13. Alberton SB, Alberton V, de Carvalho RV. Providing a harmonious smile with laminate veneers for a patient with peg-shaped lateral incisors. J Conserv Dent 2017; 20(3):210-3.

14. Patras M, Naka O, Doukoudakis S, Pissiotis A. Management of provisional restorations' deficiencies: a literature review. J Esthet Restor Dent 2012; 24(1):26.

15. Trushkowsky R, Arias DM, David S. Digital Smile Design concept delineates the final potential result of crown lengthening and porcelain veneers to correct a gummy smile. Int J Esthet Dent 2016; 11(3):338-54.

16. St-Pierre L, Cobb DS. Enhancement of aesthetic treatment planning and communication using a diagnostic mock-up. Cosmetic Dentistry 2012; 6(3):20-4.

17. Vailati F, Carciofo S. Treatment planning of adhesive additive rehabilitations: the progressive wax-up of the three-step technique. Int J Esthet Dent 2016; 11(3):356-77.

18. Regish KM, Sharma D, Prithviraj DR. Techniques of fabrication of provisional restoration: an overview. Int J Dent 2011; 2011:134659.
19. Boberick KG, Bachstein TK. 1998 Judson C. Hickey Scientific Writing Award. Use of a flexible cast for the indirect fabrication of provisional restorations. The Journal of Prosthetic Dentistry 1999; 82(1):90-3.

20. Kano P, Yoshinaga L. Skyn concept. Disponível em URL: http://www.paulokanoinstitute.com/skyn-concept.

\section{Endereço para correspondência:}

Luiz Gustavo Fernandes Lima Oliveira

Av. Lindolfo Monteiro, 813 - Fátima

CEP 64049-440, Teresina, Piauí, Brasil

Telefone: (86) 3217-3215

E-mail: luizgustavocd@hotmail.com

Recebido: 05 / 10/17. Aceito: 07/ 12 / 17. 